\title{
Bipedal Walking Control based on the Assumption of the Point-contact: Sagittal Motion Control and Stabilization
}

\author{
Tadayoshi Aoyama $^{1}$, Kosuke Sekiyama ${ }^{1}$, \\ Yasuhisa Hasegawa ${ }^{2}$ and Toshio Fukuda ${ }^{1}$ \\ ${ }^{1}$ Nagoya University, ${ }^{2}$ University of Tsukuba
}

Japan

\section{Introduction}

In the age of an aging society, the prospective role of robots is turning gradually from just working machines to do monotonous work in a factories to partners who support human life. In recent years, a lot of autonomous humanoid robots have been actually realized (Hirai et al. (1998); Kaneko et al. (2008)). These robots can walk on two legs stably by means of the control based on ZMP (Zero Moment Point). ZMP (Vukobratovic \& Borovac (2004)), the indicator of the stability of biped walking, is a point on the floor where the torque generated by both inertial and gravitational forces becomes zero. That is, using ZMP-based control to realize stable walking makes sense, thus a number of researches of ZMP-based control have been presented (Nishiwaki et al. (2002); Takanishi et al. (1985)). However, in terms of the practical use of humanoid robots, these controllers based on ZMP have a problem in terms of the runtime of the battery since ZMP-based method does not take advantage of the robot inherent dynamics.

In order to achieve natural and energy efficient biped walking, many control methods based on robot dynamics had been proposed up to this day. As one of such methods, some researchers presented the control methods to take advantage of robot dynamics directly by use of point-contact state between a robot and the ground (Furusho \& Sano (1990); Goswami et al. (1997); Grishin et al. (1994); Kuo (1999); Nakanishi et al. (2004); Ono et al. (2004)). Miura et al. produced the point-contact biped robot like stilt and realize dynamic walking by means of stabilizing control to change the configuration at foot-contact (Miura \& Shimoyama (1984)). Kajita et al. proposed the control and stabilizing method based on the conserved quantity derived by designing the COG trajectory parallel to the ground (Kajita et al. (1992)). Chevallereau presented the control to converge robot dynamics on optical trajectory by introducing the virtual time (Chevallereau (2003)). Grizzle and Westervelt et al. built the controller by use of the virtual holonomi constraint of joints named virtual constraint realize stable dynamic walking by means of the biped robot with a torso (Grizzle et al. (2001); Westervelt et al. (2004)).

As one of point-contact methods, Doi et al. proposed Passive Dynamic Autonomous Control (PDAC) previously (Doi et al. (2004b)). PDAC expresses the robot dynamics as an onedimensional autonomous system based on the two concepts: 1) point-contact 2) virtual con- 


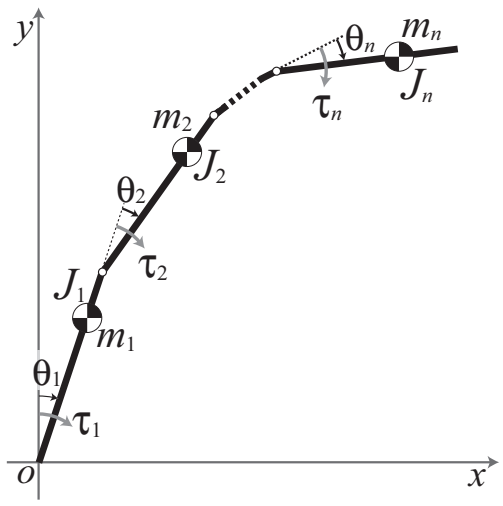

Fig. 1. Mechanical model of the serial n-link rigid robot. $\theta_{i}$ and $\tau_{i}$ are the angle and the torque of $i$ th joint respectively. $m_{i}$ and $J_{i}$ are the mass and the moment of inertia of $i$ th link respectively.

straint (proposed by Grizzle and Westervelt et al. (Grizzle et al. (2001); Westervelt et al. (2004))). In this chapter, we design the sagittal motion controller by applying PDAC to sagittal motion. In addition, we find the convergence domain of the proposed controller and prove the stability by the Liapunov Theory. Finally, 3-D dynamic walking based on the robot inherent dynamics is realized by coupling the sagittal motion proposed in this chapter and lateral motion proposed previously (Doi et al. (2004a)).

\section{Passive Dynamic Autonomous Control}

\subsection{Converged dynamics}

As mentioned previously, PDAC is base on the two concepts, i.e. point-contact and virtual constraint. Point-contact denotes that a robot contacts the ground at a point, that is, the first joint is passive. virtual constraint was defined by Grizzle and Westervelt et al. (Grizzle et al. (2001); Westervelt et al. (2004)) as a set of holonomic constraints on the robot's actuated DoF parameterized by the robot's unactuated DoF. Assuming that PDAC is applied to the serial n-link rigid robot shown in Fig. 1, these two premises are expressed as follows:

$$
\begin{aligned}
\tau_{1} & =0, \\
\boldsymbol{\Theta} & =\left[\theta_{1}, \theta_{2}, \cdots, \theta_{n}\right]^{T}=\left[f_{1}(\theta), f_{2}(\theta), \cdots, f_{n}(\theta)\right]^{T} \\
& :=\boldsymbol{f}(\theta),
\end{aligned}
$$

where $\theta$ is the angle around the contact point in the absolute coordinate system, that is, $\theta_{1}=$ $f_{1}(\theta)=\theta$.

The dynamic equations of this model are given by

$$
\frac{d}{d t}(\boldsymbol{M}(\boldsymbol{\Theta}) \dot{\boldsymbol{\Theta}})-\frac{1}{2} \frac{\partial}{\partial \boldsymbol{\Theta}}\left(\dot{\boldsymbol{\Theta}}^{T} \boldsymbol{M}(\boldsymbol{\Theta}) \dot{\boldsymbol{\Theta}}\right)-G(\boldsymbol{\Theta})=\boldsymbol{\tau},
$$

where $\boldsymbol{M}(\boldsymbol{\Theta}):=\left[\boldsymbol{m}_{1}(\boldsymbol{\Theta})^{T}, \boldsymbol{m}_{2}(\boldsymbol{\Theta})^{T}, \ldots, \boldsymbol{m}_{n}(\boldsymbol{\Theta})^{T}\right]^{T}, \boldsymbol{\Theta}:=\left[\theta_{1}, \theta_{2}, \cdots, \theta_{n}\right]^{T}$, $\boldsymbol{G}(\boldsymbol{\Theta}):=\left[G_{1}(\boldsymbol{\Theta}), G_{2}(\boldsymbol{\Theta}), \cdots, G_{n}(\boldsymbol{\Theta})\right]^{T}, \boldsymbol{\tau}:=\left[\tau_{1}, \tau_{2}, \cdots, \tau_{n}\right]^{T}, \frac{\partial}{\partial \boldsymbol{\Theta}}=\left[\frac{\partial}{\partial \theta_{1}}, \frac{\partial}{\partial \theta_{2}}, \cdots, \frac{\partial}{\partial \theta_{n}}\right]^{T}$. 
Since in this model the dynamic equation around the contact point has no term of the Coriolis force, it is given as

$$
\frac{d}{d t}\left(\boldsymbol{m}_{1}(\boldsymbol{\Theta})^{T} \dot{\boldsymbol{\Theta}}\right)-G_{1}(\boldsymbol{\Theta})=\tau_{1} .
$$

By differentiating Eq. (2) with respect to time, the following equation is acquired,

$$
\dot{\boldsymbol{\Theta}}=\frac{\partial \boldsymbol{f}(\theta)}{\partial \theta} \dot{\theta}=\left[\frac{\partial f_{1}(\theta)}{\partial \theta}, \frac{\partial f_{2}(\theta)}{\partial \theta}, \cdots, \frac{\partial f_{n}(\theta)}{\partial \theta}\right]^{T} \dot{\theta} .
$$

Substituting Eqs. (1), (2), and (5) into Eq. (3) yields the following dynamic equation,

$$
\frac{d}{d t}(M(\theta) \dot{\theta})=G(\theta)
$$

where

$$
\begin{aligned}
M(\theta) & :=\boldsymbol{m}_{1}(\boldsymbol{f}(\theta))^{T} \frac{d \boldsymbol{f}(\theta)}{d \theta} \\
G(\theta) & :=G_{1}(\boldsymbol{f}(\theta)) .
\end{aligned}
$$

By multiplying both sides of Eq. (6) by $M(\theta) \dot{\theta}$ and integrating with respect to time, the dynamics around the contact point is obtained as follows:

$$
\begin{aligned}
& \int(M(\theta) \dot{\theta}) \frac{d}{d t}(M(\theta) \dot{\theta}) d t=\int M(\theta) G(\theta) \dot{\theta} d t \\
\Longleftrightarrow & \frac{1}{2}(M(\theta) \dot{\theta})^{2}=\int M(\theta) G(\theta) d \theta .
\end{aligned}
$$

Therefore, the whole robot dynamics is expressed as the following one-dimensional autonomous system (that is, the phase around contact point),

$$
\begin{aligned}
\dot{\theta} & =\frac{1}{M(\theta)} \sqrt{2 \int M(\theta) G(\theta) d \theta} \\
& :=\frac{1}{M(\theta)} \sqrt{2(D(\theta)+C)} \\
& :=F(\theta) .
\end{aligned}
$$

In this chapter, we term Eqs. (12) and (13) Converged dynamics.

\subsection{PDAC Constant}

Since Converged dynamics is autonomous, in addition, independent of time, it is considered as a conservative system. The integral constant in right side of Eq. (10), C, is a conserved quantity, which is termed PDAC Constant. Its value is decided according to the initial condition (as for biped walking, the state just after foot-contact), and kept constant during a cycle of motion. Thus, it is possible to stabilize the motion by keeping PDAC Constant at certain value.

The dimension of PDAC Constant is equal to the square of angular momentum and has relevance to it. As is well know, assuming that the robot shown in Fig. 1 is placed on its side, the angular momentum around contact point is conserved since there is no effect of 
gravitational force on the robot dynamics. In this condition, it is clear that $M(\theta) \dot{\theta}$ (angular momentum $)=\sqrt{2 C}$ from Eq. (12), since $G(\boldsymbol{\Theta})=0$ in Eq. (3) hence $D(\theta)=0$. Note that, although angular momentum is not conserved in the condition which robot dynamics is affected by the gravitational force, PDAC Constant is conserved since it includes the effects of the gravitation. This chapter demonstrates the convergence of PDAC Constant by the Lyapunov theory and proves the stability of walking.

\section{3D biped walking}

In this section, control architecture of 3-D biped walking is summarize.

\subsection{Sagittal motion}

\subsubsection{3-link model}

For the sake of simplicity, in this chapter upper body of a robot is not moved, hence the 3-link model as shown in Fig. 2 is employed. The dynamic equation of this model is described as Eq. (3) and that of the ankle joint of the stance leg is Eq. (4) where $n=3$. The left-hand side of Eq. (4) is described as follows:

$$
\begin{aligned}
& M_{11}(\boldsymbol{\Theta})=J_{1}+J_{2}+J_{3}+m_{1} a_{1}^{2}+m_{2} l_{1}^{2}+m_{2} a_{2}^{2}-2 m_{2} a_{2} l_{1} \cos \theta_{2} \\
& +m_{3} l_{1}^{2}+m_{3} a_{3}^{2}+2 m_{3} a_{3} l_{1} \cos \left(\gamma-\theta_{3}\right), \\
& M_{12}(\boldsymbol{\Theta})=-J_{2}-m_{2} a_{2}^{2}+m_{2} a_{2} l_{1} \cos \theta_{2} \text {, } \\
& M_{13}(\boldsymbol{\Theta})=-J_{3}-m_{3} a_{3}^{2}-m_{3} a_{3} l_{1} \cos \left(\gamma-\theta_{3}\right), \\
& G_{1}(\boldsymbol{\Theta})=\left(m_{1} a_{1}+m_{2} l_{1}+m_{3} l_{1}\right) g \sin \theta_{1}+m_{2} g a_{2} \sin \left(\theta_{2}-\theta_{1}\right) \\
& +m_{3} g a_{3} \sin \left(\theta_{1}+\gamma-\theta_{3}\right) \text {, }
\end{aligned}
$$

where $\mathbf{m}_{1}(\boldsymbol{\Theta})=\left[M_{11}(\boldsymbol{\Theta}), M_{12}(\boldsymbol{\Theta}), M_{13}(\boldsymbol{\Theta})\right]$.
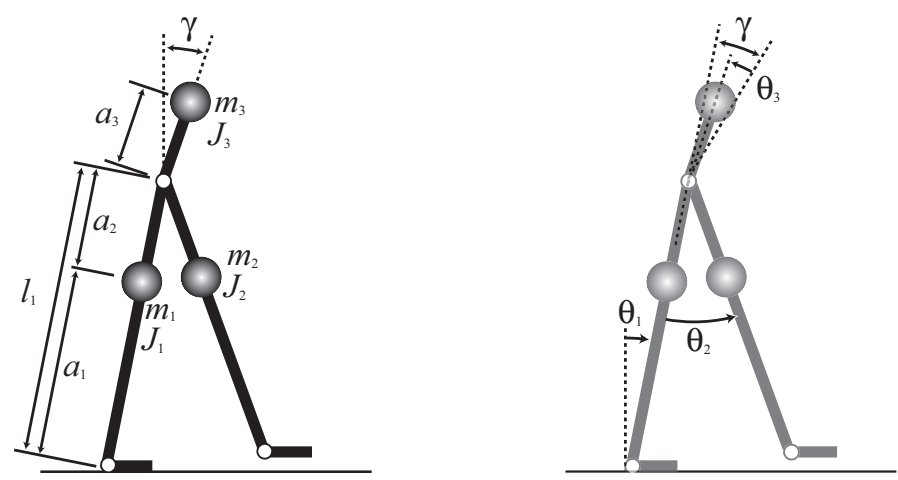

Fig. 2. 3-link model in the sagittal plane. $m_{i}, J_{i}, l_{i}$ and $a_{i}$ are the mass, the moment of inertia, the length of link and the distance from the joint to the link COG of link $i$ respectively. $\gamma$ is the angle of the forward tilting. In the right figure, $\theta_{1}, \theta_{2}$ and $\theta_{3}$ are the ankle angle of the stance leg, the angle from the stance leg to the the swing leg, the angle to swing the trunk up respectively. 


\subsubsection{Constraints of sagittal joints}

Constraints are simply designed as follows:

- The angle of the torso is constant.

- The swing leg is symmetrical to the stance leg.

That is,

$$
\begin{aligned}
& \theta_{1}=f_{1}(\theta)=\theta, \\
& \theta_{2}=f_{2}(\theta)=2 \theta, \quad \text { and } \\
& \theta_{3}=f_{3}(\theta)=\theta .
\end{aligned}
$$

From Eqs. (14)-(16) and (1), Eq. (6) is

$$
\begin{aligned}
M_{S}(\theta) & =J_{1}-J_{2}+m_{1} a_{1}^{2}+m_{2} l_{1}^{2}-m_{2} a_{2}^{2}+m_{3} l_{1}^{2}+m_{3} a_{3} l_{1} \cos (\gamma-\theta) \\
& :=E_{1}+E_{2} \cos (\gamma-\theta), \\
G_{S}(\theta) & =\left(m_{1} a_{1}+m_{2} l_{1}+m_{2} a_{2}+m_{3} l_{1}\right) g \sin \theta+m_{3} g a_{3} \sin \gamma \\
& :=E_{3}+E_{4} \sin \theta .
\end{aligned}
$$

Thus,

$$
\begin{aligned}
\int M_{S}(\theta) G_{S}(\theta) d \theta \\
=\quad \int\left(E_{1}+E_{2} \cos (\gamma-\theta)\right)\left(E_{3}+E_{4} \sin \theta\right) d \theta \\
=\quad E_{2} E_{4}\left(\frac{\sin \gamma}{2} \theta-\frac{\cos (2 \theta-\gamma)}{4}\right)+E_{1} E_{3} \theta \\
\quad \quad+E_{2} E_{3} \sin (\theta-\gamma)-E_{1} E_{4} \cos \theta+C_{s} \\
\quad=\quad D_{s}(\theta)+C_{s},
\end{aligned}
$$

where $C_{s}$ is the integral constant, which is PDAC Constant of the sagittal motion. From Eq. (12), Converged dynamics in the sagittal plane is

$$
\begin{aligned}
\dot{\theta} & =\frac{1}{M_{S}(\theta)} \sqrt{2\left(D_{s}(\theta)+C_{s}\right)} \\
& :=F_{S}(\theta) .
\end{aligned}
$$

Note that it is necessary that $\gamma$ is decided so that $M_{\mathcal{S}}(\theta)>0$ in order to avoid singular point. Generally speaking, as for humanoid robots and biped robots, $E_{1}>E_{2}$ since $l_{1}>a_{3}$. Thus, we assume $M_{\mathcal{S}}(\theta)>0$ below.

\subsubsection{Foot-contact model}

Regarding foot-contact, it is assumed that the ground is perfectly inelastic collision and occurred for a moment similarly to previous works (Goswami et al. (1997); Grizzle et al. (2001); Kuo (1999); Westervelt et al. (2004)). That is, the angular momentum around the contact point is conserved before and after foot-contact.

Fig. 3 shows the angle and length of the inverted pendulum at foot-contact. Here, consider the foot-contact at the end of $k$ th step, i.e. at the beginning of $k+1$ th step. Denoting the angular 

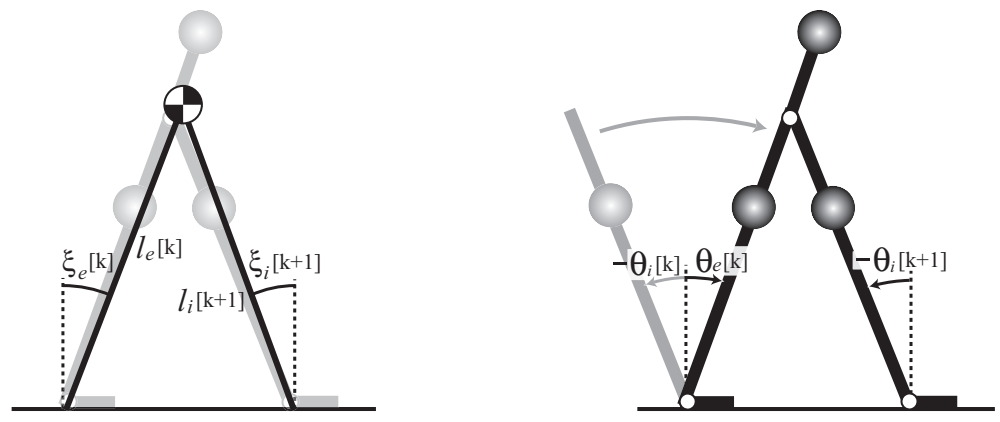

Fig. 3. Parameters at foot-contact. $l_{e}[k]$ and $\xi_{e}[k]$ are the length and inclination of the inverted pendulum which connects the ankle of support leg and robot COG before impact at the end of $k$ th step. $l_{i}[k+1]$ and $\xi_{i}[k+1]$ are those after impact. $\theta_{e}[k]$ and $\theta_{i}[k+1]$ are the angles around the contact point before and after impact.

velocity of ankle joint of the rear leg at foot-contact as $\dot{\theta}_{e}[k]$, the following equation is derived from Eq. (12):

$$
\dot{\theta}_{e}[k]=\frac{1}{M_{S}\left(\theta_{e}[k]\right)} \sqrt{2\left(D_{s}\left(\theta_{e}[k]\right)+C_{s}[k]\right)},
$$

where $C_{S}[k]$ denotes PDAC Constant of $k$ th step.

Since the torso angle is constant and COG is not rotated, the angular velocity of ankle joint of the fore leg at foot-contact, $P_{i}[k+1]$, is described as follows:

$$
\begin{aligned}
P_{i}[k+1] & =m_{t} l_{e}[k] l_{i}[k+1] \dot{\theta}_{e}[k] \cos \left(\xi_{e}[k]+\xi_{i}[k+1]\right) \\
& =\frac{m_{t} l_{e}[k] l_{i}[k+1] \cos \left(\xi_{e}[k]+\xi_{i}[k+1]\right)}{M_{s}\left(\theta_{e}[k]\right)} \sqrt{2\left(D_{s}\left(\theta_{e}[k]\right)+C_{s}[k]\right)} \\
& :=h[k] \sqrt{2\left(D_{s}\left(\theta_{e}[k]\right)+C_{s}[k]\right)},
\end{aligned}
$$

where $m_{t}=m_{1}+m_{2}+m_{3}$.

Since the angular velocity around the passive joint is

$$
P=M_{s}(\theta) \dot{\theta},
$$

PDAC Constant after foot-contact, $C_{S}[k+1]$, is represented as

$$
\begin{aligned}
C_{s}[k+1] & =\frac{1}{2} P_{i}[k+1]^{2}-D_{s}\left(\theta_{i}[k]\right) \\
& =h[k]^{2} C_{s}[k]+h[k]^{2} D_{s}\left(\theta_{e}[k]\right)-D_{s}\left(\theta_{i}[k]\right) \\
& :=s_{1}[k] C_{s}[k]+s_{2}[k] .
\end{aligned}
$$




\subsubsection{Sagittal motion period}

In order to satisfy the condition of constant step-length, it is necessary to control the lateral motion so that lateral foot-contact period matches sagittal one. Since sagittal dynamics is expressed as an one-dimensional autonomous dynamics, it is possible to calculate the sagittal foot-contact period by integrating sagittal Converged dynamics with time as follows:

$$
T_{S}=\int_{i \theta^{+}}^{i+1} \frac{\theta^{-}}{F_{S}(\theta)} d \theta
$$

In next section, we design the lateral motion and build the controller satisfying the synchronization between lateral and sagittal motion.

\subsection{Lateral motion control}

\subsubsection{Lateral motion}

In this section, the lateral motion is composed by means of PDAC. In phase(A), a robot starts to turn over toward its swing-leg-side and is accelerated by gravitation from the tilting position at a standstill on the stance-leg-side to foot-contact. In phase(B), after foot-contact, a robot is got up toward the tilting position at a standstill by the energy obtained in phase(A).

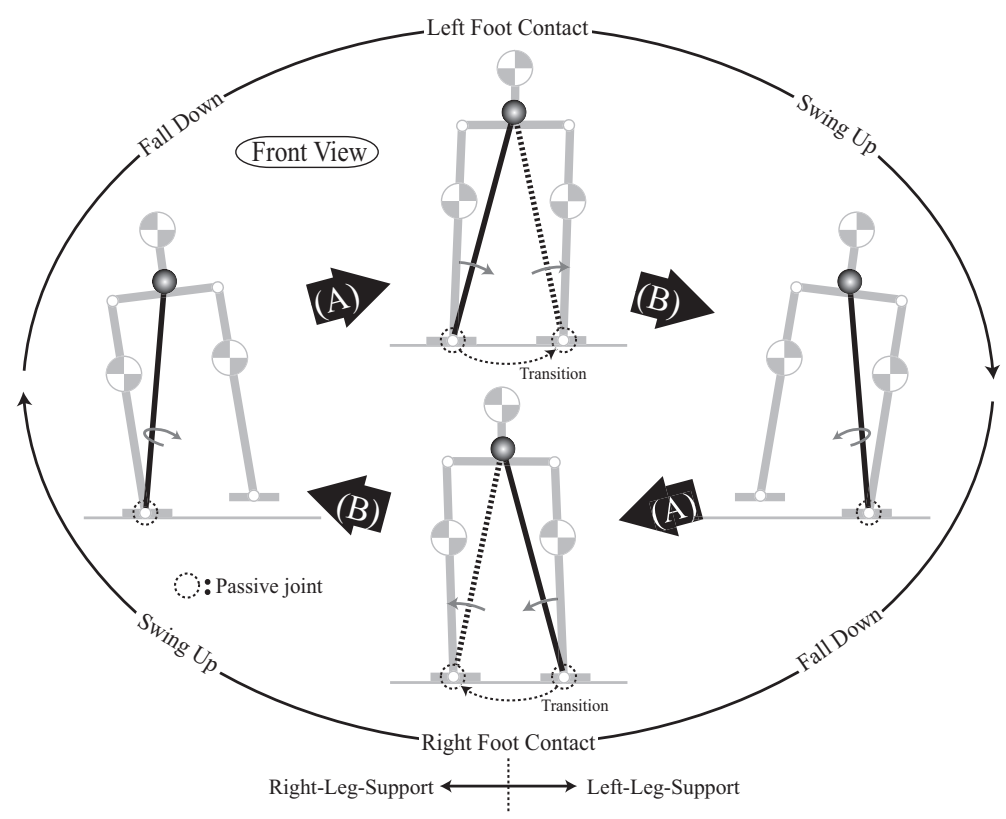

Fig. 4. The lateral motion of lateral-based walk (front view). The inverted pendulum falls off in phase(A) and swing up in phase(B) 


\subsubsection{Constraint of lateral joints}

The dynamic equation of an inverted pendulum is described as follows:

$$
\frac{d}{d t}\left(\left(m l^{2}+J\right) \dot{\phi}\right)=m g l \sin \phi \text {. }
$$

Multiplying both side of this equation by $\left(m l^{2}+J\right) \dot{\phi}$ and integrating with respect to time yields the following equations,

$$
\begin{aligned}
& \left(\left(m l^{2}+J\right) \dot{\phi}\right) \frac{d}{d t}\left(\left(m l^{2}+J\right) \dot{\phi}\right)=m g l\left(m l^{2}+J\right) \dot{\phi} \sin \phi \\
\Longleftrightarrow & \frac{1}{2}\left(\left(m l^{2}+J\right) \dot{\phi}\right)^{2}=\int m g l\left(m l^{2}+J\right) \dot{\phi} \sin \phi d t \\
\Longleftrightarrow & \dot{\phi}=\frac{1}{m l^{2}+J} \sqrt{2 \int m g l\left(m l^{2}+J\right) \dot{\phi} \sin \phi d t .}
\end{aligned}
$$

Here, we decide the virtual constraint, that is, pendulum length $l$ is described as the function of $\phi$. It is clear that the right side of Eq. (27) can be integrated if $f(\phi)$ is a polynomial equation. Thus in this chapter, $f(\phi)$ is decided as follows:

$$
\begin{aligned}
l & =f(\phi) \\
& =a \phi^{2}+b \phi+c,
\end{aligned}
$$

where $a, b$, and $c$ are determined so as to satisfy the conditions described below. At first, the conditions of pendulum length at the beginning and ending of phase(A) and phase(B) introduce the following four equations:

$$
\begin{aligned}
& f^{A}\left(\phi_{0}\right)=l_{0}, \\
& f^{A}\left(\phi_{1}\right)=l_{0}+\Delta l, \\
& f^{B}\left(-\phi_{2}\right)=l_{0}-\Delta l, \\
& f^{B}\left(-\phi_{3}\right)=l_{0},
\end{aligned}
$$

where upper-suffixes denote the differentiation of phases.

In addition, the pendulum motion is designed so that the angular velocity of robot joints is not discontinuous, that is, the velocity along pendulum is zero,

$$
\begin{aligned}
& \frac{\partial f^{A}}{\partial \phi}\left(\phi_{1}\right)=0, \quad \text { and } \\
& \frac{\partial f^{B}}{\partial \phi}\left(-\phi_{2}\right)=0 .
\end{aligned}
$$

From Eq. (30)-(35), $a$ and $b, c$ in each phase are decided.

Finally, the phase around contact point (phase of passive joint) is obtained from Eqs. (27) and (28) as follows:

$$
\begin{aligned}
\dot{\phi} & =\frac{1}{m f(\phi)^{2}+J} \sqrt{2 \int m g f(\phi)\left(m f(\phi)^{2}+J\right) \sin d \phi} \\
& :=\frac{1}{M_{l}(\phi)} \sqrt{2\left(D_{l}(\phi)+C_{l}\right)} \\
& :=F(\phi) .
\end{aligned}
$$


Lastly, the value to lift up pelvis, $\Delta l$, is determined. $\Delta l$ is necessary to be decided so that Eq. (36) satisfies the initial condition of phase $(\mathrm{A})$ and the end condition of phase(B), that is,

$$
\begin{aligned}
& F_{l}^{A}\left(\phi_{0}\right)=F_{l}^{B}\left(-\phi_{3}\right)=0 \\
\Longleftrightarrow & \frac{\sqrt{2\left(D_{l}^{A}\left(\phi_{1}\right)-D_{l}^{A}\left(\phi_{0}\right)\right)}}{M_{l}^{A}\left(\phi_{1}\right)} \cos \left(\phi_{1}+\phi_{2}\right)=\frac{\sqrt{2\left(D_{l}^{B}\left(-\phi_{2}\right)-D_{l}^{B}\left(-\phi_{3}\right)\right)}}{M_{l}^{B}\left(-\phi_{2}\right)},
\end{aligned}
$$

where upper suffixes denote the differentiation of phases. $\Delta l$ is so small that it is possible to find the appropriate value satisfying Eq. (37) by use of the quadratic approximation.

\subsubsection{Control of lateral period}

Next we design the period controller of the lateral motion described in the previous subsection. The period of lateral motion is decided by the amplitude of pendulum motion, that is, the period is long if the amplitude is large and it is short if the amplitude is small. In this chapter, the desired period is realized by controlling the lateral amplitude.

Assuming that the pendulum angle at the transition from phase(B) to phase(A) is $\phi_{3}$, the motion period $T$ can be found properly by the following calculation

$$
\int_{-\phi_{3}}^{-\phi_{2}} \frac{1}{F_{B}(\phi)} d \phi+\int_{\phi_{3}}^{\phi_{1}} \frac{1}{F_{A}(\phi)} d \phi=T .
$$

However, it is not easy to solve this equation for $\phi_{3}$. The pendulum extension is so small that the desired amplitude is decided approximately by use of the model of inverted pendulum, length of which is not variable, as follows:

$$
\phi_{3}=\frac{\phi_{c}}{\cosh \left(\sqrt{\frac{g}{l_{0}}} \frac{T}{2}\right)},
$$

where, $\phi_{c}$ is the pendulum angle in the standing posture, i.e. the pendulum angle at the footcontact under the condition of $\Delta l=0$.

\section{Stability proof of the sagittal motion}

\subsection{Constraint of constant step-length}

In this work, the step-length is fixed at constant value in order to stabilize walking. Under such condition, it is clear that the following is held:

$$
\theta_{e}[j]=-\theta_{i}[j]=\arcsin \left(\frac{\lambda_{d}}{2 l_{1}}\right):=\theta_{c}=\text { const., }
$$

where $j \in \mathbf{N}$ and $0 \leq \theta_{c}<\frac{\pi}{2}$. Since the torso angle, $\gamma$, is kept constant, $\xi_{e}[j]$ and $\xi_{i}[j], l_{e}[j], l_{i}[j]$ are also all constant similarly. Hence, in Eq. (23),

$$
h[j]:=H=\text { const. }
$$

is held. Besides, in Eq. (24),

$$
\begin{aligned}
s_{1}[j] & =h[j]^{2}=H^{2}:=S_{1}=\text { const. } \\
s_{2}[j] & =h[j]^{2} D_{s}\left(\theta_{e}[j]\right)-D_{s}\left(\theta_{i}[j]\right) \\
& =H^{2} D_{s}\left(\theta_{c}\right)-D_{s}\left(-\theta_{c}\right):=S_{2}=\text { const. }
\end{aligned}
$$


are also held.

\subsection{Requisite to perform walking continuously}

We consider the requisite to generate walking. In terms of practicality, let $\gamma$ be $0<\gamma<\frac{\pi}{2}$. In order to perform walking continuously, $\dot{\theta}>0$ is required at all times. Considering $M_{\mathcal{S}}(\theta)>0$, this condition is equivalent to $P=M_{\mathcal{S}}(\theta) \dot{\theta}>0$. Since

$$
\begin{aligned}
\frac{d D_{s}(\theta)}{d \theta} & =M_{s}(\theta) G_{s}(\theta) \\
& =\left(E_{1}+E_{2} \cos (\gamma-\theta)\right)\left(E_{3}+E_{4} \sin \theta\right),
\end{aligned}
$$

and also since $E_{3}>0$ and $E_{4}>0$, in can be seen that $D_{s}(\theta)$ i.e. the angular velocity of passive joint, $P$, is minimum when

$$
\theta=\arcsin \left(-\frac{E_{3}}{E_{4}}\right):=\hat{\theta}
$$

and that it decreases monotonically on $-\frac{\pi}{2}<\theta<\hat{\theta}$ and increases monotonically on $\hat{\theta}<\theta<$ $\frac{\pi}{2}$. Since $\frac{1}{2} P^{2}=D_{S}(\theta)+C_{S}$, the condition discussed above, $P>0$, is described as below,

$$
P>0 \Longleftrightarrow C_{s}>-D_{s}(\hat{\theta}):=\hat{C}_{s} .
$$

Therefore, from Eq. (24), the requisite to perform walking continuously is found as follows:

$$
S_{1} \hat{C}_{s}+S_{2}>\hat{C}_{s} .
$$

Next, we argue the state that dynamics of walking is converged on a unique trajectory, i.e. the equilibrium state. On such condition, PDAC Constant of every step is converged on constant value. That is,

$$
C_{s}[k]=C_{s}[k+1]:=C_{S}^{*} .
$$

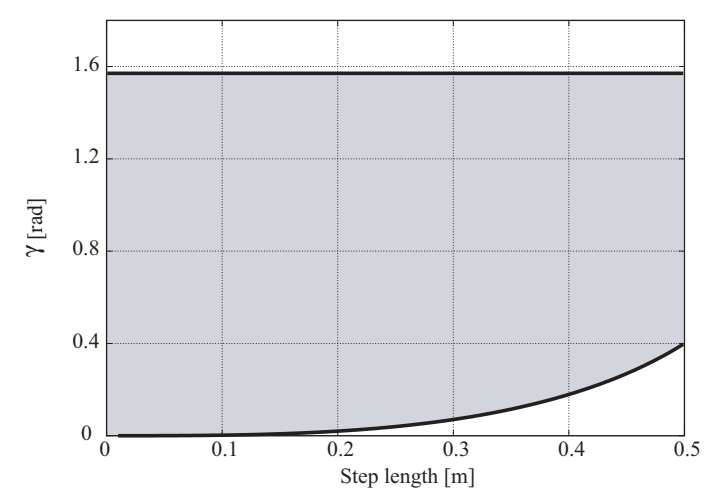

Fig. 5. Condition of $\theta$ and $\gamma$ in order to generate continual walking 
Hence, from Eq. (24),

$$
\begin{aligned}
& C_{s}^{*}=S_{1} C_{s}^{*}+S_{2} \\
& \Longleftrightarrow S_{2}=C_{s}^{*}\left(1-S_{1}\right)
\end{aligned}
$$

is held. Substituting Eq. (43) into Eq. (42),

$$
\left(1-S_{1}\right)\left(C_{s}^{*}-\hat{C}_{s}\right)>0
$$

is obtained. From Eq. (41), it is clear that

$$
C_{s}^{*}>\hat{C}_{s} .
$$

From Eqs. (44) and (45), the requisite to perform walking continuously, Eq. (42), is described as follows:

$$
1-S_{1}>0 \text {. }
$$

As for our robot (Gorilla Robot III (Kajima et al. (2004)): Fig. 9, Table 1), Eq. (46) is the range shown in Fig. 5. Although we assume $\gamma<\frac{\pi}{2}$ in Fig. 5, actual upper bound is decided according to the limitation of robot's specification such as the torque to swing a leg forward or to keep a torso angle at constant value.

\subsection{Proof of stability}

Lastly, we prove the stability by Liapunov Theory using the conditions found above. The error between actual $C_{S}$ and convergent value is defined as

$$
\delta C_{s}:=C_{s}^{*}-C_{s} .
$$

The following positive definite function, $V$, is defined,

$$
V=\left(\delta C_{s}\right)^{2} \text {. }
$$

Since $V(0)=0$ and $V>0\left(\delta C_{s} \neq 0\right)$, it is apparent that $V$ is positive definite. From Eqs. (24), (43), and (47), finite difference of $V$ is

$$
\begin{aligned}
& \Delta V=V[k+1]-V[k] \\
& =\delta C_{s}[k+1]^{2}-\delta C_{s}[k]^{2} \\
& =\left(\delta C_{s}[k+1]+\delta C_{s}[k]\right)\left(\delta C_{s}[k+1]-\delta C_{s}[k]\right) \\
& =-\left(2 C_{s}^{*}-C_{s}[k+1]-C_{s}[k]\right)\left(C_{S}[k+1]-C_{S}[k]\right) \\
& =-\left(2 C_{s}^{*}-S_{1} C_{s}[k]-S_{2}-C_{s}[k]\right)\left(S_{1} C_{s}[k]+S_{2}-C_{s}[k]\right) \\
& =-\left(2 C_{s}^{*}-S_{1} C_{s}[k]-\left(1-S_{1}\right) C_{s}^{*}-C_{s}[k]\right)\left(S_{1} C_{s}[k]+\left(1-S_{1}\right) C_{s}^{*}-C_{s}[k]\right) \\
& =-\left(1+S_{1}\right)\left(1-S_{1}\right)\left(C_{s}^{*}-C_{s}[k]\right)^{2} \\
& =-\left(1+S_{1}\right)\left(1-S_{1}\right) \Delta C_{S}[k]^{2} \text {. }
\end{aligned}
$$

Since it is clear that $1+S_{1}=1+H^{2}>0$ from Eq. (41) and that $1-S_{1}>0$ from Eqs. (46) and (48) is

$$
\Delta V=0 \quad\left(\delta C_{s}=0\right)
$$

In addition,

$$
\Delta V<0 \quad\left(\delta C_{s} \neq 0\right)
$$

is held. From Eq. (49) and (50), $\Delta V$ is negative definite. Therefore, the equilibrium point, $C_{S}^{*}$, is asymptotically stable in the range shown in Fig. 5. 


\subsection{Simulation}

Next, stability proof described in the previous subsection is confirmed by the numerical simulation. Fig. 6 is the phase portrait of $\theta$ and alteration in terms of time. From these figures, the convergence of the sagittal motion can be ascertained. On this simulation, step-length is $0.18[\mathrm{~m}]$, the torso angle is $\gamma=0.035$ [rad].

Fig. 7 depicts the return maps of PDAC Constant and the angular velocity of passive joint at foot-contact. These figures show that the sagittal dynamics has a sole stable fixed point.

Finally, in order to confirm that the stability of the sagittal motion is independent of steplength, we perform the simulation of the various step-length. Fig. 8 is the graph of the return map of the angular volocity of passive joint at foot-contact v.s. step-length. From this figure, it can be confirmed that the sagittal dynamics is stable regardless of step-length.
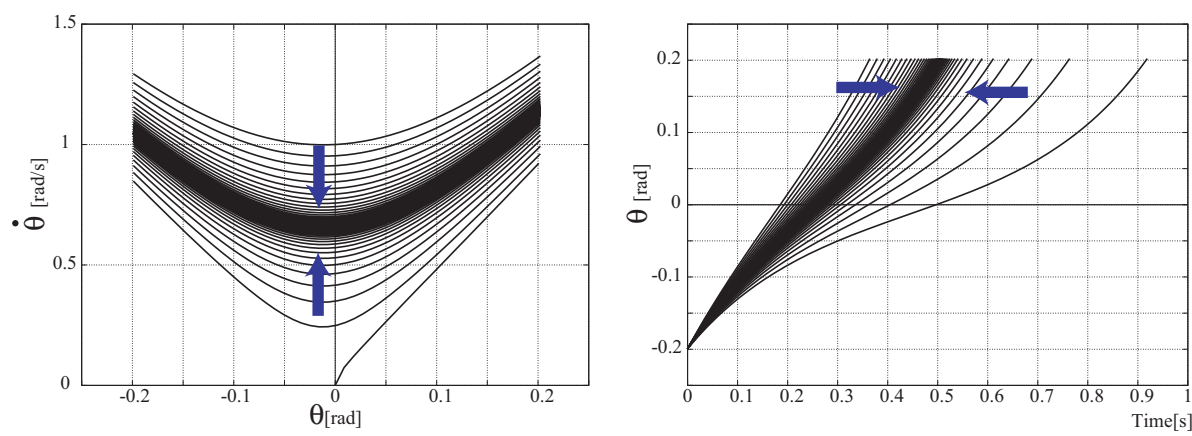

Fig. 6. Simulation results of sagittal stabilization. (Left) Phase portrait of the sagittal dynamics. (Right) $\theta$ trajectory vs. time.
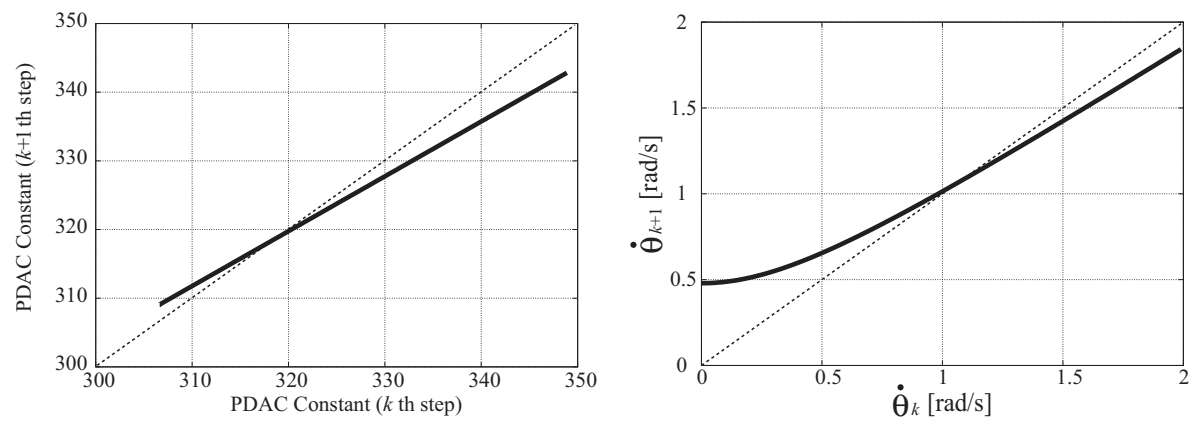

Fig. 7. Simulation results of sagittal stabilization. (Left) Return map of PDAC Constant. (Right) Return map of $\dot{\theta}$ at foot-contact. 


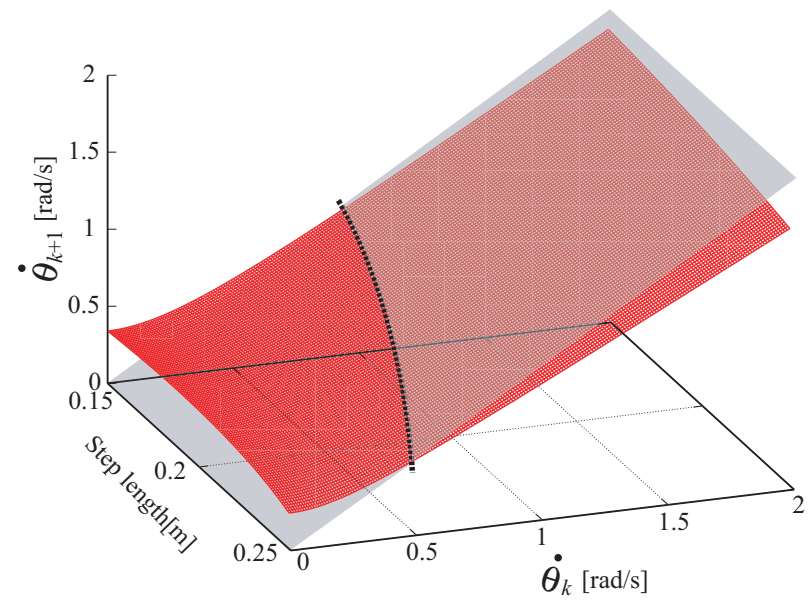

Fig. 8. Return map of $\dot{\theta}$ at foot-contact vs. step-length

Table 1. Link parameters of Gorilla Robot III

\begin{tabular}{l|c|c|c}
\hline Mass[kg] & link1 & $m_{1}$ & 2.618 \\
\hline & link2 & $m_{2}$ & 3.451 \\
\hline & link3 & $m_{3}$ & 15.143 \\
\hline COG position & link1 & $a_{1}$ & 0.23 \\
\hline & link2 & $a_{2}$ & 0.28 \\
\hline & link3 & $a_{3}$ & 0.22 \\
\hline Moment of inertia & link1 & $J_{1}$ & 0.042 \\
\hline & link2 & $J_{2}$ & 0.070 \\
\hline & link3 & $J_{3}$ & 0.047 \\
\hline
\end{tabular}
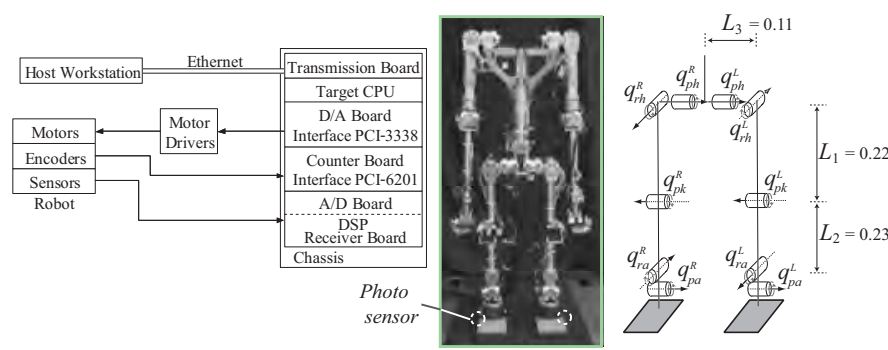

Fig. 9. Gorilla Robot III (about 1.0[m] height, 22.0[kg] weight, $24 \mathrm{DOF}$ ) 


\section{Experiment}

In order to ascertain the validity of proposed method, we conducted the experiment. Both sagittal controller and lateral one are employed simultaneously and 3-D dynamic walking is realized. Note that, in the following experiment, estimated viscous torque is applied to the ankle joints of stance-leg in order to realize the passive joint virtually.

\subsection{Experimental Setup}

Fig. 9 depicts our experimental setup and our robot "Gorilla Robot III (Kajima et al. (2004))". This robot is driven by 24 AC motors of $20-30$ W with 100-200 times of speed reduction by harmonic gears. It has the photo sensors on its soles to perceive foot-contact.

\subsection{Experimental results}

In this experiment, the robot bends its knee joint of the swing leg so as to prevent the foot being in friction with the ground immediately after foot-contact on the assumption that the effect of knee bending on the robot dynamics can be neglected. The foot of the swing leg is actuated so as to be kept parallel to the ground. The experiment was performed on the basically level and flat ground which has maximum $1.0[\mathrm{~cm}]$ irregularity without discontinuities.

In this chapter, the desired step-length is given to be gradually increased within initial 5 steps up to $0.15[\mathrm{~m}]$ and after 5 steps it is fixed at $0.15[\mathrm{~m}]$. As a result of experiment, the dynamic and natural walking is realized over 25 steps. The step-length is about $0.15[\mathrm{~m}]$ and the walking velocity is about $0.23[\mathrm{~m} / \mathrm{s}]$. Fig. 10 shows the snapshots of the PDAC walking at 1st, 5th, 9th, 12th, 14th, 16th step respectively. The angle and angular velocity of the lower body joints are depicted in Fig. 11 and Fig. 12. As shown in these figures, the smooth dynamics motion is realized periodically.

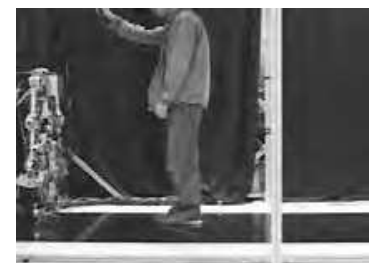

(a)

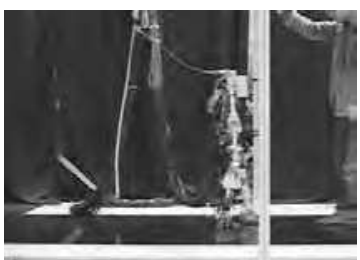

(d)

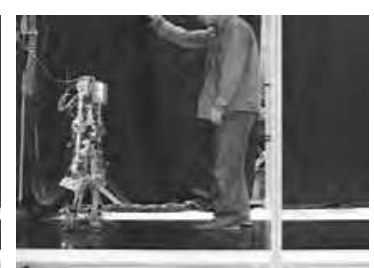

(b)

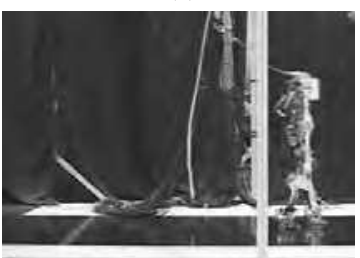

(e)

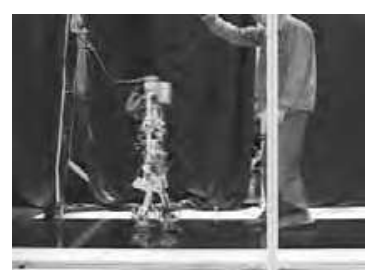

(c)

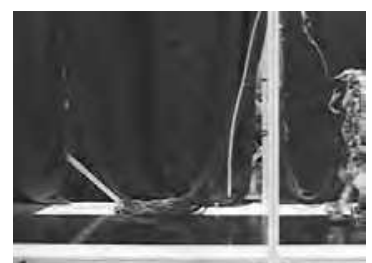

(f)

Fig. 10. Snapshots of the walking experiment. Each figure shows the snapshots at (a)1st (b)5th (c) 9 th (d)12th (e)14th (f)16th step. 


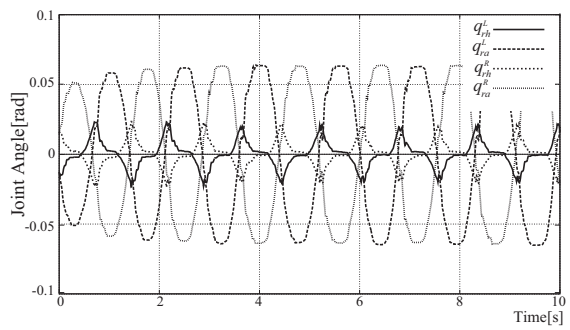

(a)

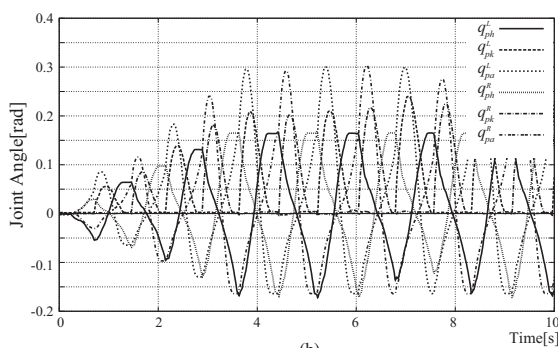

Fig. 11. Angle of the joints (a) in the lateral plane (b) in the sagittal plnae
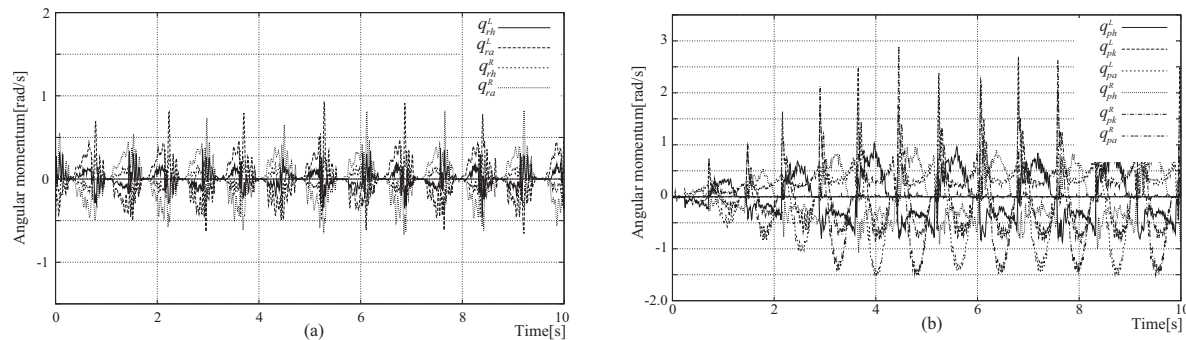

Fig. 12. Angular Velocity of the joints (a) in the lateral plane (b) in the sagittal plnae

\section{Conclusion}

This chapter designed the sagittal motion controller of biped walking and proved the stability of sagittal motion designed by means of PDAC. The proof was conducted by the Liapunov Theory, and the convergence domain was also investigated. We confirmed the correctness of proof by numerical simulation. Finally, a three-dimensional dynamic walking whose steplength is about $0.15[\mathrm{~m}]$ and velocity is about $0.23[\mathrm{~m} / \mathrm{s}]$ was realized by coupling the sagittal motion and lateral motion. The proof in this chapter handled the stability of 2-D dynamics, thus the future work is to propose the walking controller achieving the stability of 3-D dynamics, and prove the 3-D stability of dynamic walking.

\section{References}

Chevallereau, C. (2003). Time-Scaling Control for an Underactuated Biped Robot. IEEE Transactions on Robotics Automation, Vol.19, No.2, pp.362-368, 2003.

Doi, M.; Hasegawa, Y. \& Fukuda, T. (2004a). Passive trajectory control of the lateral motion in bipedal walking. Proceedings of IEEE International Conference on Robotics and Automation, pp.3049-3054, New Orleans, May 2004.

Doi, M.; Hasegawa, Y. \& Fukuda, T. (2004b). Passive Dynamic Autonomous Control of Bipedal Walking. Proceedings of IEEE-RAS/RSJ International Conference on Humanoid Robots, Paper no.72, Los Angeles, November 2004.

Furusho, J. \& Sano, A. (1990). Sensor-based Control of a Nine-Link Biped. The International Journal of Robotics Research, Vol.9, No.2, pp.83-98, 1990. 
Goswami, A.; Espiau, B. \& Keramane, A. (1997). Limit cycles in a passive compass gait biped and passivity-mimicking control laws. Autonomous Robots, Vol.4, No.3, pp.273-286, 1997.

Grishin, A. A.; Formal'sky, A. M.; Lensky, A. V. \& Zhitomirsky, S. V. (1994). Dynamic Walking of a Vehicle With Two Telescopic Legs Controlled by Two Drives. The International Journal of Robotics Research, Vol.13, No.2, pp.137-147, 1994.

Grizzle, J. W.; Abba, G. \& Plestan, F. (2001). Asymptotically Stable Walking for Biped Robots: Analysis via Systems with Impulse Effects. IEEE Transactions on Automatic Control, Vol.46, No.1, pp.56-64, 2001.

Hirai, K.; Hirose, M.; Haikawa, Y. \& Takenaka, T. (1998).The Development of Honda Humanoid Robot. Proceedings of the IEEE International Conference on Robotics and Automation, pp.1321-1326, Leuven, May 1998.

Kajima, H.; Doi, M.; Hasegawa, Y. \& Fukuda, T. (2004). A study on brachiation controller for a multi-locomotion robot -realization of smooth, continuous brachiation-. Advanced Robotics, vol.18, no.10, pp.1025-1038, 2004.

Kajita, S.; Yamaura, T. \& Kobayashi, A. (1992). Dynamic walking control of a biped robot along a potential conserving orbit. IEEE Transactions on Robotics Automation, Vol.8, No.4, pp.431-438, 1992.

Kaneko, K.; Harada, K.; Kanehiro, F.; Miyamori, G. \& Akachi, K. (2008). Humanoid Robot HRP-3. Proceedings of the IEEE/RSJ International Conference on Intelligent Robots and Systems, pp.2471-2478, Nice, September 2008.

Kuo, A. D. (1999). Stabilization of Lateral Motion in Passive Dynamic Walking. The International Journal of Robotics Research, Vol.18, No.9, pp.917-930, 1999.

Miura, H. \& Shimoyama, I., (1984). Dynamic Walking of a biped. The International Journal of Robotics Research, Vol.3, No.2, pp.60-74, 1984.

Nakanishi, J.; Morimoto, J.; Endo, G.; Cheng, G.; Schaal, S. \& Kawato, M. (2004). A Framework for Learning Biped Locomotion with Dynamical Movement Primitives, Proceedings of IEEE-RAS/RSJ International Conference on Humanoid Robots, Paper no.81, Los Angeles, November 2004.

Nishiwaki, K.; Kagami, S.; Kuniyoshi, Y.; Inaba, M. \& Inoue, H. (2002). Online Generation of Humanoid Walking Motion based on a Fast Generation Method of Motion Pattern that Follows Desired ZMP. Proceedings of the International Conference on Intelligent Robots and Systems, pp.2684-2689, Lausanne, September 2002.

Ono, K.; Furuichi, T. \& Takahashi, R. (2004). Self-Excited Walking of a Biped Mechanism With Feet. The International Journal of Robotics Research, Vol.23, No.1, pp.55-68, 2004.

Takanishi, A.; Ishida, M.; Yamazaki, Y. \& Kato, I. (1985). The Realization of Dynamic Walking Robot WL-10RD. Proceedings of International Conference on Advanced Robotics, pp.459466, Tokyo, September 1985.

Vukobratovic, M. \& Borovac, B. (2004). ZERO-MOMENT POINT-THIRTY FIVE YEARS OF ITS LIFE. International Journal of Humanoid Robotics, vol.1, no.1, pp.157-173, 2004.

Westervelt, E. R.; Buche, G. \& Grizzle, J. W. (2004). Experimental Validation of a Framework for the Design of Controllers that Induce Stable Walking in Planar Bipeds. The International Journal of Robotics Research, Vol.24, No.6, pp.559-582, 2004. 


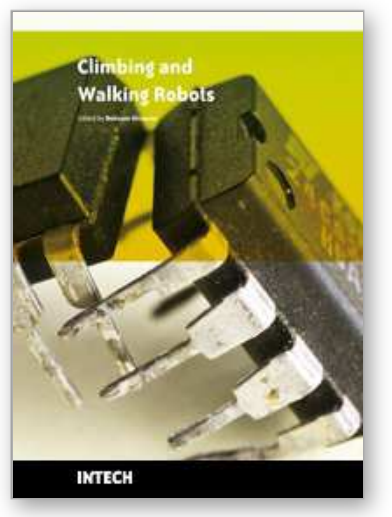

\author{
Climbing and Walking Robots \\ Edited by Behnam Miripour
}

ISBN 978-953-307-030-8

Hard cover, 508 pages

Publisher InTech

Published online 01, March, 2010

Published in print edition March, 2010

Nowadays robotics is one of the most dynamic fields of scientific researches. The shift of robotics researches from manufacturing to services applications is clear. During the last decades interest in studying climbing and walking robots has been increased. This increasing interest has been in many areas that most important ones of them are: mechanics, electronics, medical engineering, cybernetics, controls, and computers. Today's climbing and walking robots are a combination of manipulative, perceptive, communicative, and cognitive abilities and they are capable of performing many tasks in industrial and non- industrial environments. Surveillance, planetary exploration, emergence rescue operations, reconnaissance, petrochemical applications, construction, entertainment, personal services, intervention in severe environments, transportation, medical and etc are some applications from a very diverse application fields of climbing and walking robots. By great progress in this area of robotics it is anticipated that next generation climbing and walking robots will enhance lives and will change the way the human works, thinks and makes decisions. This book presents the state of the art achievments, recent developments, applications and future challenges of climbing and walking robots. These are presented in 24 chapters by authors throughtot the world The book serves as a reference especially for the researchers who are interested in mobile robots. It also is useful for industrial engineers and graduate students in advanced study.

\title{
How to reference
}

In order to correctly reference this scholarly work, feel free to copy and paste the following:

Tadayoshi Aoyama, Kosuke Sekiyama, Yasuhisa Hasegawa and Toshio Fukuda (2010). Bipedal Walking Control Based on the Assumption of the Point-Contact: Sagittal Motion Control and Stabilization, Climbing and Walking Robots, Behnam Miripour (Ed.), ISBN: 978-953-307-030-8, InTech, Available from: http://www.intechopen.com/books/climbing-and-walking-robots/bipedal-walking-control-based-on-theassumption-of-the-point-contact-sagittal-motion-control-and-sta

\section{INTECH}

open science | open minds

\section{InTech Europe}

University Campus STeP Ri

Slavka Krautzeka 83/A

51000 Rijeka, Croatia

Phone: +385 (51) 770447

\section{InTech China}

Unit 405, Office Block, Hotel Equatorial Shanghai

No.65, Yan An Road (West), Shanghai, 200040, China

中国上海市延安西路65号上海国际贵都大饭店办公楼 405 单元

Phone: +86-21-62489820 
Fax: +385 (51) 686166

Fax: +86-21-62489821

www.intechopen.com 
(C) 2010 The Author(s). Licensee IntechOpen. This chapter is distributed under the terms of the Creative Commons Attribution-NonCommercialShareAlike-3.0 License, which permits use, distribution and reproduction for non-commercial purposes, provided the original is properly cited and derivative works building on this content are distributed under the same license. 